\title{
Extracting Words and Multi-part Symbols in Graphics Rich Documents
}

\author{
Mark Burge ${ }^{\star}$ and Gladys Monagan \\ Institut für Informationssysteme \\ Swiss Federal Institute of Technology (ETH) \\ ETH-Zentrum, CH-8092 Zurich, Switzerland
}

\begin{abstract}
We present an algorithm for grouping multipart symbols, dashed lines, and character strings for extraction from line drawings. The image undergoes a lossless raster-to-vector conversion creating as its vector representation an undirected graph, a so-called run graph. Next, the image elements of the run graph are extracted and classified probabilistically based upon their geometric features using a decision tree. An area Voronoi tessellation of the members of the sets is constructed, from which a neighborhood graph is derived, which is guaranteed to be minimal and complete. The graph is then traversed to group the members of the various sets for extraction and input to different recognition modules. No a priori font or other domain specific information is required for the grouping, and no special geometrical relationships among the elements are assumed. Results are presented with example images taken from those used by our Swiss cadastral map understanding system.
\end{abstract}

\section{Introduction}

Line drawings contain semantically meaningful objects which are often made up of several visually disjoint parts, or image elements. Analyzing the elements of these objects in isolation may lead to an incorrect interpretation of the object. Such is the case when analyzing a single dash of a dashed line, and the dash in the letter " $\mathrm{i}$ ", instead of considering the entire dashed line or the complete glyph for the letter " $i$ ". We propose an algorithm which uses a neighborhood graph to represent explicitly the spatial relations between all the elements of an image. Using this graph and simple geometric features, we show that it is possible to group image elements into semantically meaningful objects without recourse to application specific knowledge.

The grouping for extraction of symbols, dashed lines, and character strings which appear in a document is a problem encountered in interpreting line images such as maps and engineering drawings [1]. Nakamura et al. [2] give five reasons why character string extraction is difficult in topographic maps: characters often touch background figures, existence of many character like figures, various orientations of strings, intra-character spacing is different from string to string, and character strings are often close together. These problems are present in many types of line drawings, not just topographic maps. The algorithm presented in this paper deals with all but one of the problems listed by

\footnotetext{
^ Now with Systemtheorie, Johannes Kepler Universität, A-4040 Linz, Austria
} 
Nakamura et al., that of characters which touch background figures. To resolve this, high level knowledge is required, which our algorithm specifically avoids using.

We present a two phase algorithm, the first phase we call localization, and the second phase grouping. (Another name for localization is segmentation, namely, the separation of text and graphics.) The algorithm does not require a priori knowledge of the font, point size, or intra-character spacing of the character strings. Descriptions of the symbols used or of the dashed line patterns are also unnecessary. The only information necessary is the resolution at which the image was scanned, and the feature values which can distinguish between the image elements which are to be localized.

\section{Localization}

Input is a raster image scanned at any resolution, which undergoes lossless raster-tovector conversion. The result of this vectorization is a graph, the run graph [3], with subgraphs which correspond to the connected components, or image elements. Using the run graph representation as the basis, geometric features are computed which are used by a decision tree to classify probabilistically each element of the image into one of five sets: dots, circles, dashes, symbols, and graphics. The features used are based on a convex hull formed from the nodes of the run graph. Features used are the area of this convex hull, the longest side and the complexity, i.e. the number of nodes in the run graph of the image element.

\section{Grouping}

Conceptually we wish to group together image elements which when combined form an object which has some semantic meaning. An example would be glyphs of various fonts which when taken together form a character string, or dashes which when grouped together form a dashed line or dashed curve. In fact, if the grouping is not done at this level, serious irreparable errors could occur. Such is the case when multi-part symbols are not grouped. A circle within a circle in a cadastral map signifies a "main point." If the circles are not grouped into one symbol, they will be interpreted independently as "marking rocks" which is one of the interpretation of single circles.

The ambiguity which arises during localization, for example between dashes, ones, and "i" $i$ "s is resolved by referring to the neighborhood graph for contextual information. In our implementation, the neighborhood graph is constructed from the area Voronoi tessellation.

The point based Voronoi tessellation is unsuited for line image understanding since the useful primitives (in the context of document understanding) are not individual pixels corresponding to geometric points, but groups of pixels corresponding to segments, arcs, ellipses, or polygonal objects. To produce the neighborhood graph, we have developed an approximated area based Voronoi tessellation which can be calculated efficiently [4].

Given that $A_{1}, A_{2}, \ldots A_{n}$ are image elements and that $p$ and $q$ are particular image elements, then we can define the distance, $d_{a}\left(p, A_{i}\right)$, from $p$ to $A_{i}$ as: $d_{a}\left(p, A_{i}\right)=$ $\min _{q \in A_{i}} d(p, q)$ where this represents the minimum Euclidean distance from $p$ to any 


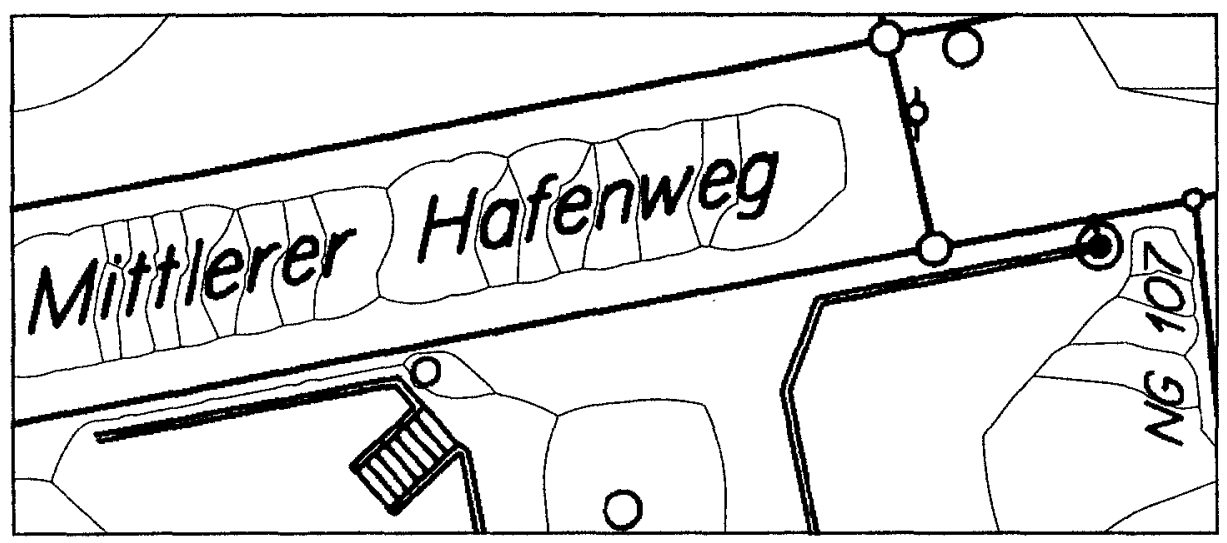

Fig. 1. Area Voronoi tessellation.

location in $A_{i}$. Using this definition [5], one can formulate the area based Voronoi tessellation as $V_{a}\left(A_{i}\right)$, where this represents the region in which the distance to $A_{i}$ is less than or equal to the distance to any other area:

$$
V_{a}\left(A_{i}\right)=p \mid d_{a}\left(p, A_{i}\right) \leq d_{a}\left(p, A_{j}\right), j \neq i, j=1, \ldots, n .
$$

In the neighborhood graph, each node represents an element of the image, and each edge a connection to its neighboring element, as defined in Equation 1. Since the neighborhood graph is derived from the Voronoi tessellation, the neighborhoods represented by the graph can be shown to be both minimal in the number of neighbors and complete. Minimal in the sense that only those image elements which are closest are included, and complete in the sense of including all of the closest image elements.

This guarantee of the neighborhood graph being minimal and complete is important so as not to lose information or introduce false information. Other methods like radial search may result in incomplete neighborhoods, and methods like Wahl's [6] run length smearing and Meng's [7] bounding box extents may result in neighborhoods which are neither complete nor minimal. These methods can still group because they rely on additional knowledge (assumptions) about the relative positions of the elements.

The goal of the grouping process is to search the neighborhood graph to generate subgraphs so that the members of the set of dots, set of symbols, and set of dashes can be combined into candidate character strings, candidate multipart symbols, and dashed lines. When grouping, the proximity of the image elements is important but not a sufficient condition to enforce grouping. For example, in a labeled graphic, a given character may be closer to the graphic then it is to the other characters in the label; yet, this character should be grouped with the other characters into a label and not with the graphic. We want to group image elements that are similar to each other; thus we consider as local features for the grouping, both descriptive features of the image elements, as well as proximity. 
The search for the grouping is as follows:

1. traverse the neighborhood graph in a breadth first manner

- calculate the local neighborhood features

2. traverse the graph in a depth first manner

- join edges into a subgraph, depending on the local neighborhood features

3. traverse the subgraphs in a breadth first search

- adaptively recalculate the local thresholds to try to extend the subgraph

- repeat until no more changes occur

The candidate dash lines are then passed on to the vectorization module, whereas the candidate multipart symbols and the candidate strings need to be interpreted by the symbol recognizer and/or word recognizer.
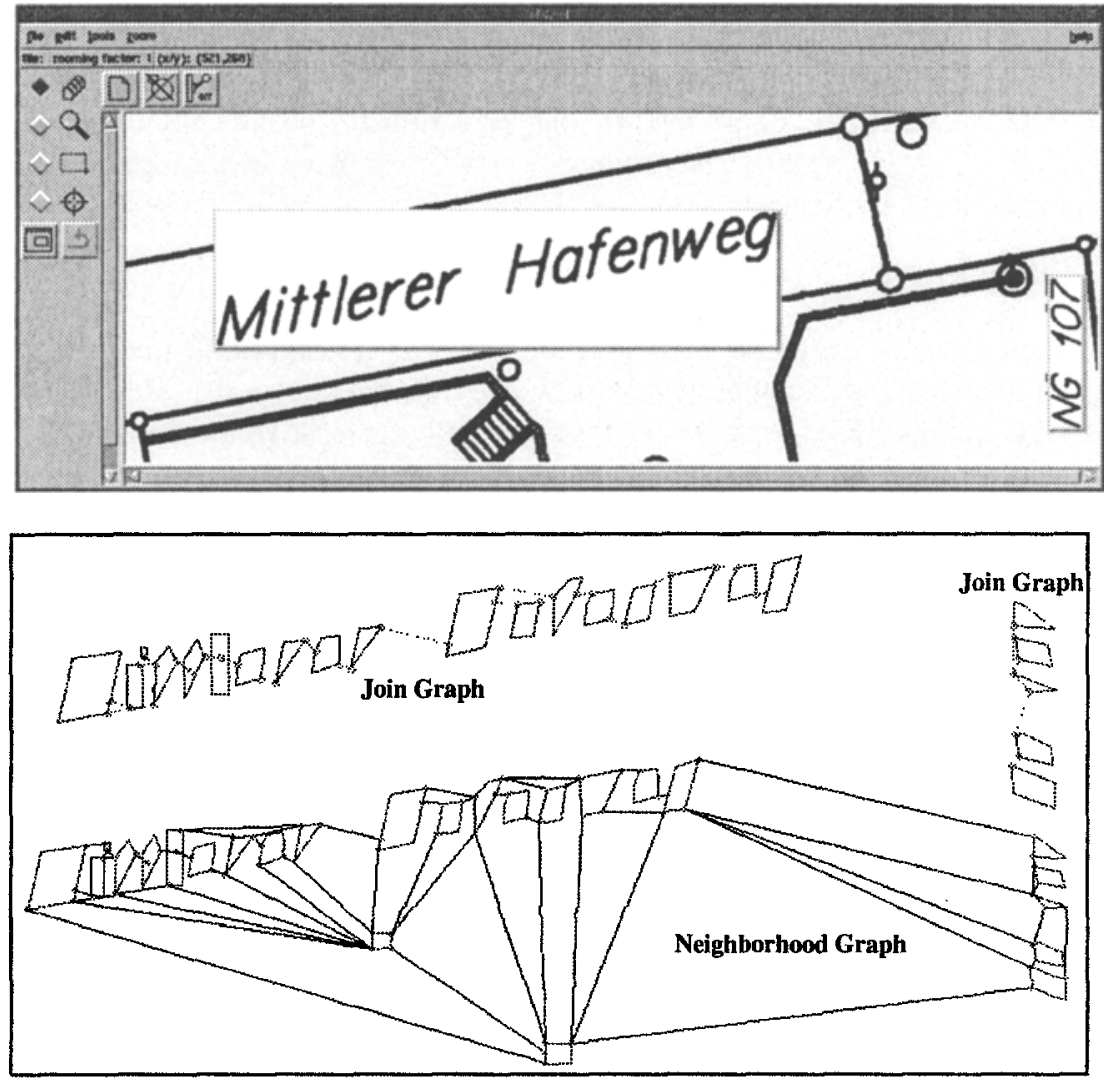

Fig. 2. In the top part of the figure, two strings which were grouped: they are written on bitmaps which are placed on top of the original raster image. Underneath, the two subgraphs resulting from the search graph algorithm. Underneath these, the neighborhood graph from which the subgraphs were derived. 


\section{Experimental Results}
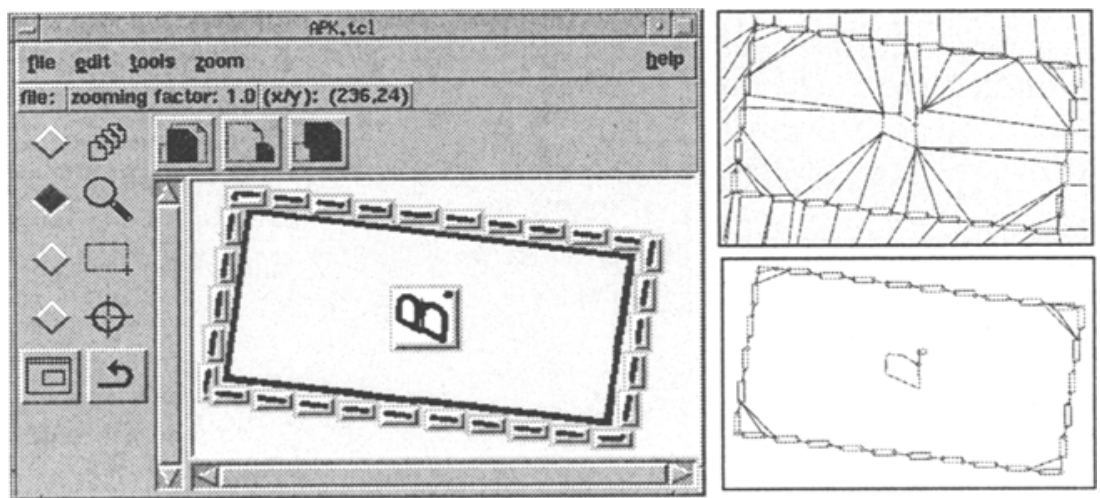

Fig. 3. On the left, a grouped dashed line and a grouped character string. On the top right the corresponding neighborhood graph and on the bottom right its subgraphs.

Figure 2 and Figure 3 show the results of our algorithm as used in our system for understanding Swiss cadastral maps. Table 1 below summarizes performance times for some sample images. The entry components is the number of image elements in the image, image size is given in pixels, localization is the number of seconds for the first phase including: reading the file, raster-to-vector conversion, feature calculation, and CART classification. Grouping includes the time for constructing the area Voronoi tessellation and the graph search for grouping. All tests were conducted on a Sparc 10 with $48 \mathrm{MB}$ of real memory using algorithms implemented in $\mathrm{C}++$.

\begin{tabular}{|l|c|c|c|c|}
\hline \multicolumn{1}{|c|}{ name } & components & image size & localization & grouping \\
\hline Duden [8] document image & 155 & $447 \times 271$ & $1.3 \mathrm{~s}$ & $7.3 \mathrm{~s}$ \\
\hline CAD image & 47 & $1386 \times 1741$ & $3.4 \mathrm{~s}$ & $13.3 \mathrm{~s}$ \\
\hline Mittlerer example & 153 & $3556 \times 1052$ & $5.7 \mathrm{~s}$ & $30.7 \mathrm{~s}$ \\
\hline cadastral map A0 & 2829 & $13584 \times 19019$ & $86.1 \mathrm{~s}$ & $844.1 \mathrm{~s}$ \\
\hline
\end{tabular}

Table 1. Performance of localization and grouping.

\section{Conclusions}

After an image has been scanned, the image elements have to be localized and grouped so that they can be processed by higher level recognition modules. The main advantages 
that we see for the presented method of localization is that no a priori font or other domain specific information is required, and when grouping image elements, minimal and complete neighborhoods are examined. To state again, there is no predetermined number of elements in a neighborhood (e.g. K-nearest neighbors) nor is there a fixed maximum or minimum distance between the elements of a group (e.g. inter-character gap), and lastly, no fixed requirements about the geometry of the elements (e.g. that they all lie along a line) are assumed.

The authors thank the financial support of the Swiss Federal Commission for the Advancement of Scientific Research (KWF), project 2540.1, and of the Aargauisches Elektrizitätswerk (AEW). AEW also provided us with most of the test data used here. The work presented here was done at the ETH, Zürich.

\section{References}

1. L. A. Fletcher and R. Kasturi, "A Robust Algorithm for Text String Separation from Mixed Text/Graphics Images," IEEE Transactions of Pattern Analysis and Machine Intelligence, vol. 10, pp. 910-918, Nov. 1988.

2. A. Nakamura, O. Shiku, M. Anegawa, C. Nakamura, and H. Kuroda, "A Method for Recognizing Character Strings from Maps Using Linguistic Knowledge," in Proc. of the Second International Conf. on Document Analysis and Recognition, (Tsukuba, Japan), pp. 561-564, IEEE Computer Society Press, Oct. 20-22 1993.

3. L. Boatto et al., "An Interpretation System for Land Register Maps," IEEE Computer, vol. 25, pp. 25-34, July 1992.

4. M. Burge and G. Monagan, "Using the Voronoi tessellation for grouping words and multi-part symbols in documents, "in Proc. VISION GEOMETRY IV, SPIE's International Symposium on Optics, Imaging and Instrumentation, Vol. 2573, (San Diego, California), July 9-14 1995.

5. A. Okabe, B. Boots, and K. Sugihara, "Nearest neighbourhood operations with generalized Voronoi diagrams: a review," International Journal of Geographical Information Systems, vol. 8, pp. 43-71, January-February 1994.

6. F. Wahl et al., "Block Segmentation and Text Extraction in Mixed Text/Image Documents," Computer Vision, Graphics and Image Processing, vol. 20, pp. 375-390, 1982.

7. L. Meng, "Toward the Automatic Digitization of Map Text," in Mustererkennung 1991, Proc. 13. DAGM Symposium (B. Radig, ed.), pp. 361-366, Springer Verlag, Oct. 1991.

8. W. R. der Dudenredaktion: G. Drosdowski, ed., Der Duden: in 12 Bänden; das Standardwerk zur deutschen Sprache. Dudenverlag, 1992. 